Research Article

\title{
Selective Consecutive Determination of Desloratadine and Montelukast Sodium in Their Pure and Binary Dosage Form Based on Pencil Graphite Electrochemical Sensors
}

\author{
Amir Alhaj Sakur $(\mathbb{D}$, Dania Nashed $(\mathbb{D}$, and Imad Noureldin $(\mathbb{D}$ \\ Analytical and Food Chemistry Department, Faculty of Pharmacy Aleppo University, Aleppo, Syria \\ Correspondence should be addressed to Amir Alhaj Sakur; profsakur@gmail.com,Dania Nashed; dania.nashed92@gmail.com, and \\ Imad Noureldin; imadnoureldin55@yahoo.com
}

Received 23 February 2021; Revised 25 March 2021; Accepted 15 April 2021; Published 5 May 2021

Academic Editor: Larisa Lvova

Copyright (c) 2021 Amir Alhaj Sakur et al. This is an open access article distributed under the Creative Commons Attribution License, which permits unrestricted use, distribution, and reproduction in any medium, provided the original work is properly cited.

\begin{abstract}
In this study, we present a new, green electrochemical method for potentiometric estimation of desloratadine and montelukast sodium in their pure and binary dosage form. For that, three pencil graphite sensors were fabricated; the first one was prepared to analyse desloratadine drug (DES) by coating the graphite bar with the coating membrane, which comprises the ion pair of desloratadine and ammonium reineckate reagent (RNK), the polymer poly vinyl chloride (PVC), and the plasticizers dibutyl phthalate (DBP). The second one, which was used to analyse montelukast (MON), was constructed by using the ion pair of cadmium chloride reagent (Cd.) with montelukast and the same earlier named polymer and plasticizer. As a trial to analyse both of the drugs by the same sensor consecutively, we have constructed a combined pencil graphite electrode, which contains the two earlier suggested ion pairs, that is, we can use this electrode to selectively analyse for each drug. The proposed electrodes were effectively used for analysis of DES and MON as a single dosage form and as combined pharmaceutical preparation, without any need for prior separation that was performed depending on the difference in the efficient $\mathrm{pH}$ range for each sensor. The proposed sensors exhibited a Nernstian equation slopes of $-30.11,27.70,(-29.16,29.79) \mathrm{mv}$. decade ${ }^{-1}$ in the linearity range $5.00 \times 10^{-5}-1.00 \times 10^{-2}$ and $1.00 \times 10^{-5}-1.00 \times 10^{-2} \mathrm{M}$, respectively. The sensors exhibit high sensitivity according to LOD values $((0.036-0.018)-(0.025-0.026) \mu \mathrm{M})$, respectively, and important selectivity toward the studied drugs in presence of interfering ions and excipients. The optimum circumstances were studied, and the method was validated by application of ICH rules. Finally, the method was compared with a documented method, and the required statistical values were calculated.
\end{abstract}

\section{Introduction}

In the last few years, there has been an urgent need to design new combined dosage forms to enhance the efficacy or reduce the side effects of the drugs. That demands develop new analytical techniques, which can analyse these combinations in a fast, simple, precise way [1].

Desloratadine (DES), 8-chloro-6,11-dihydro-11-(4piperidylidene) $-5 \mathrm{H}$ benzo $[2,3]$ cyclohepta $[1,2-b]$ pyridine [4] (Figure 1), is a nonsedating antihistamine used in the symptomatic release of allergic conditions such as rhinitis and urticaria [5]. The literature review revealed that several methods are available for the determination of DES individually including $\operatorname{HPLC}[2,6]$, spectrophotometric $[3,7]$, and voltammetric [8]. However, there is not any potentiometric method to determine DES before this study.

Montelukast sodium is (1-(1R)-1-[3-[(1E)-2-(7-chloro2-quinolinyl) ethenyl] phenyl]-3-[2-(1-hydroxy-1-methylethyl) phenyl]-propyl] thio] methyl) cyclopropane acetic acid [4] (Figure 2). It is a cysteinyl leukotriene receptor antagonist and used for treatment of asthma [9]. Some research studies were reported to determine MON as individual drug such as HPLC [10, 11], spectrophotometric $[12,13]$, and potentiometric [14-16].

The combination of desloratadine and montelukast sodium significantly improved nasal symptoms, and it is used 
<smiles>Clc1ccc2c(c1)CCc1cccnc1C2=C1CCNCC1</smiles>

FIGURE 1: Desloratadine structure.

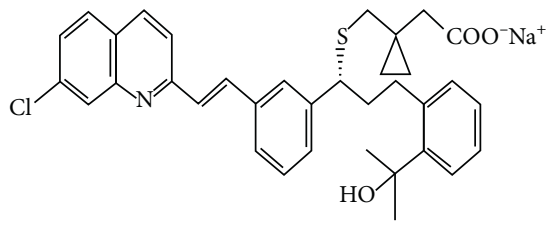

FIGURE 2: Montelukast sodium structure.

for treatment of asthma, allergies, and chronic urticaria [17]. Some reported methods have been used for the simultaneous determination of desloratadine and montelukast such as HPLC $[18,19]$ and spectrophotometry $[20,21]$. However, no electrochemical method is mentioned in the literature to determine DES and MON as a combined form. This promoted us to develop a new simple, sensitive, selective, rapid, and green potentiometric technique using a pencil graphite sensor for the analysis of the two drugs in their coformulated tablets. Electrochemical methods have a lot of advantages compared with the other analytical methods because of their selectivity, sensitivity, simplicity, and the wide linear range which allow the analyst to determine drugs and ions in small concentrations [22-26]. Among all the electrochemical techniques, pencil graphite electrodes have many features such as the small size, their fast response time, and the long lifetime $[14,27]$ compared to those classical ion-selective electrodes [28-31]. The main purpose of the study is to draw attention to the ability of electrochemical methods for quantitative estimation of drugs in their combination forms competitively with the other common analytical techniques used to determine combination forms such as HPLC and spectrophotometric methods, which were considered as complex, timeconsuming, and organic solvent-consuming methods.

\section{Materials and Methods}

2.1. Chemicals. All used solvents and materials were analytical class; high pure desloratadine and montelukast sodium, polyvinyl chloride (PVC), tetrahydrofuran (THF), and dibutyl phthalate (DBP) were purchased from SigmaAldrich, Germany.

Ammonium reineckate and cadmium chloride were purchased from BDH Chemicals, England.

Bidistilled water was used to prepare solutions.

\subsection{Apparatus}

Radiometer analytical-ion check $10 \mathrm{pH} / \mathrm{mv}$ meter (Cedex, France)

\author{
Crison pH meter model Glp21/EU (Spain) \\ Ultrasonic bath-Powersonic 405 (Korea) \\ Sartorius balance model 2474 (Germany)
}

\subsection{Standard Solutions}

2.3.1. Standard Solution of DES. A stock solution of DES $\left(1.00 \times 10^{-2} \mathrm{M}\right)$ was made by dissolving exact weight equivalent to $0.155 \mathrm{~g}$ of drug powder in a $50 \mathrm{ml}$ volumetric flask using bidistilled water as a solvent; then, a series of working solutions, their concentrations varying $1.00 \times 10^{-7}-1.00 \times 10^{-3} \mathrm{M}$, were prepared by continuing dilutions from the first stock solution.

2.3.2. Standard Solution of MON. A stock solution of MON $\left(1.00 \times 10^{-2} \mathrm{M}\right)$ was made by dissolving exact weight equivalent to $0.304 \mathrm{~g}$ of drug powder in a $50 \mathrm{ml}$ volumetric flask using bidistilled water as a solvent; then, a series of working solutions, their concentrations varying $1.00 \times 10^{-7}-1.00 \times 10^{-3} \mathrm{M}$, were prepared.

\subsection{Pharmaceutical Formulations}

Aerius (5 mg F.C. Tab). Each tablet is claimed to contain $5 \mathrm{mg}$ of DES, and it was manufactured by Unipharma pharmaceutical company (Syria).

Azmalir (10 mg F.C. Tab). Each tablet is claimed to contain $10 \mathrm{mg}$ of $\mathrm{MON}$, and it was manufactured by Unipharma pharmaceutical company (Syria).

Desolate-M Tab. Each tablet contains $5 \mathrm{mg}$ DES and $10 \mathrm{mg} \mathrm{MON}$, and it was manufactured by Archicare Limited (India).

\subsection{Procedure}

2.5.1. Fabrication of the Sensors. The first step to fabricate the sensor is to prepare the ion pairs of drugs and reagents. For the DES graphite sensor, $1 \mathrm{mmol}$ of DES was mixed with $1 \mathrm{mmol}$ of ammonium reineckate, and a pink precipitate was formed. For the MON graphite sensor, $1 \mathrm{mmol}$ of MON was mixed by $1 \mathrm{mmol}$ of cadmium chloride, and a yellow precipitate was formed. Then, the precipitates were filtered and washed several times by bidistilled water to be used later as an electroactive material in the coating solutions for the two electrodes separately [16].

The second step is to prepare the coating solutions, $0.6 \mathrm{~g}$ of PVC with $1.2 \mathrm{~g}$ of DBP; then, we added $0.2 \mathrm{~g}$ of IP (DES and RNK in case of sensor 1 and MON and Cd. in case of sensor 2) for the combined sensor, $0.48 \mathrm{~g}$ PVC with $0.96 \mathrm{~g}$ DBP; then, we added $0.2 \mathrm{~g}$ of each IP (DES.RNK and MON.Cd.). Then, all the components were dissolved in a small volume of THF. The last step is to fabricate the pencilcoated graphite electrodes, performed by immersing the end of a graphite rod ( $2 \mathrm{~mm}$ in diameter) in the previous coating solutions several times to get a suitable thickness of polymeric film that was required [32]. Each electrode was 
activated before the measurement of the potential by dipping it in $1.00 \times 10^{-3} \mathrm{M}$ of drug solution for $24 \mathrm{hrs}$.

2.5.2. Sensors Calibration. The fabricated sensors were immersed in junction with the $\mathrm{Ag} / \mathrm{AgCl}$ reference electrode in standard series solutions of DES or MON $\left(1 \times 10^{-6}-1 \times 10^{-2} \mathrm{M}\right)$ separately. The potential produced by the suggested electrodes was read for each concentration. Calibration graphs were constructed related to the electrode potential values versus the negative logarithmic value of the drug concentration [33].

\subsubsection{Potential Measurement Conditions of the Proposed} Sensors. We have studied some of the conditions that may have an important effect on the electrode potential; these conditions are the effect of components percentage, the effect of $\mathrm{pH}$ and temperature, response time, and the selectivity of the electrode in existence of several obstructive ions and excipients.

2.5.4. Preparation of the Sample's Solutions. The proposed sensors were used for the determination of desloratadine and montelukast sodium in some pharmaceutical preparations as single and combined dosage forms. Twenty tablets of each medication were softly powdered; precise weight proportionate to one tablet was taken, dissolved, and sonicated in the ultrasonic bath for 5 minutes and filtered. An exact volume was taken from the filtrate and diluted to $25 \mathrm{ml}$ to get $10^{-4} \mathrm{M}$ of drug solution.

\section{Results and Discussion}

In this study, we determine each of DES and MON in their pure and combined form, depending on the idea that DES acts as a cation in which it makes up an ion pair with reineckate anion and MON acts as an anion in which it makes up an ion pair with cadmium cation. We can determine each drug using its proposed sensor without any obstruction from the other drug potentials, depending on the difference in the active $\mathrm{pH}$ range for each sensor, so that we were able to determine each of these drugs in their binary mixture by applying an accurate, precise, sensitive method, which presents a new potentiometric method to analyse DES and MON combination, instead of other sophisticated analytical methods reported to analyse this combination.

\subsection{Performance Characteristics of the Developed Sensors.} The constructed sensors in conjunction with the $\mathrm{Ag} / \mathrm{Ag} \mathrm{Cl}$ reference electrode were used for the direct determination of DES and MON in their standard series solutions, and their concentrations range $1 \times 10^{-6}-1 \times 10^{-2} \mathrm{M}$. Calibration slope for each sensor was measured from day to day and found to be almost stable over a period of 63 days for the DES graphite sensor and of 49 days for the MON graphite sensor. The performance characteristics of the proposed graphite sensors are given in Table 1.
3.2. Effect of the Percentage of Coating Solution's Components. The coating solution, which was used as a coating film covering the pencil graphite electrode, consists of the polymer (PVC), plasticizer (DBP), and the electroactive material $\left(\mathrm{IP}_{\mathrm{S}}\right)$. We have tried different percentages of these components to get the best sensor's characteristics, as given in Table 2.

3.3. Effect of $p H$ and Temperature. To determine the active $\mathrm{pH}$ range for each sensor, two concentrations $\left(1 \times 10^{-4} \mathrm{M}\right.$ and $1 \times 10^{-3} \mathrm{M}$ ) of each drug were studied separately over $\mathrm{pH}$ range $2-11$ using $\mathrm{NaOH}$ and $\mathrm{HCl} 0.1 \mathrm{M}$ to adjust the $\mathrm{pH}$ value. The potential was measured and plotted versus $\mathrm{pH}$ values for each drug using its proposed sensor. As shown in Figures 3 and 4 , we have found that the effective $\mathrm{pH}$ range was 2.5-5 for the DES.RNK sensor; at $\mathrm{PH}$ values more than 5 , the sensor's potential was unstable and unbalanced. For MON.Cd. sensor, the effective $\mathrm{pH}$ range was 6-10; at $\mathrm{pH}$ value less than 6 , the drug transferred to the unionized form which led to instability in the response.

The influence of the temperature on the sensors' response was studied in the range of temperatures $10-50^{\circ} \mathrm{C}$. We have found a gradual increase in electrodes' potential as the temperature increases. Calibration graphs at each temperature value were plotted, and we found that the slope values remain almost stable over temperatures range $10-50^{\circ} \mathrm{C}$, which indicates the thermal stability of the constructed electrodes up to $50^{\circ} \mathrm{C}$ (Figures 5 and 6). At temperature values more than $50^{\circ} \mathrm{C}$, an obvious decrease in the slope value was found and that maybe due to the instability of the ion pairs in high temperature values.

3.4. Selectivity. The selectivity of the constructed sensors towards the drug's ion was studied using the matched potential method [34]. Furthermore, we studied the selectivity of the DES.RNK sensor toward desloratadine in presence of montelukast drug and the selectivity of MON.Cd. toward MON in presence of DES drug, and the results exhibit high selectivity as given in Table 3 .

3.5. Response Time and Reversibility. The response time of the constructed pencil graphite sensors was estimated by immersing the electrodes in the series of drug solutions, each has a 10 -fold increase in concentrations $5 \times 10^{-5}, 5 \times 10^{-4}$, and $5 \times 10^{-3} \mathrm{M}$, and measuring the average time needed to reach a steady potential within $\pm 0.1 \mathrm{mV}$ [33]. As noticed from Figures 7 and 8 , the time required to get a constant potential of the final equilibrium value is less than $20 \mathrm{~s}$.

To estimate the reversibility of the proposed graphite sensors, we recorded the potential response of 3 drug concentrations which are within the linearity range $\left(5 \times 10^{-5}\right.$, $5 \times 10^{-4}$, and $5 \times 10^{-3} \mathrm{M}$ ) from the lowest concentration to the highest and vice versa [35]. We found that the response was reversible as seen in Figures 7 and 8, but we noticed that time required to achieve the equilibrium in potential response was much higher in case of changing concentrations from the highest to the lowest. [33]. 
TABLE 1: The performance characteristics of the proposed described sensors.

\begin{tabular}{|c|c|c|c|c|}
\hline \multirow{2}{*}{ Parameter } & \multirow{2}{*}{ DES.RNK graphite sensor } & \multirow{2}{*}{ MON.Cd. graphite sensor } & \multicolumn{2}{|c|}{ Combined sensor } \\
\hline & & & DES & MON \\
\hline Slope $\left(\mathrm{mV}\right.$. decade $\left.^{-1}\right)$ & -30.11 & 27.70 & -29.16 & 29.79 \\
\hline Intercept & 345.93 & -10.17 & 337.60 & -119.73 \\
\hline Linearity range $(\mathrm{M})$ & $5.00 \times 10^{-5}-1.00 \times 10^{-2}$ & $1.00 \times 10^{-5}-1.00 \times 10^{-2}$ & $1.00 \times 10^{-5}-1.00 \times 10^{-2}$ & $5.00 \times 10^{-6}-1.00 \times 10^{-2}$ \\
\hline Correlation coefficient & 0.9996 & 0.9997 & 0.9998 & 0.9992 \\
\hline $\operatorname{LOD}^{\mathrm{a}}(\mu \mathrm{M})$ & 0.036 & 0.018 & 0.025 & 0.025 \\
\hline LOQ $(\mu \mathrm{M})$ & 0.109 & 0.055 & 0.075 & 0.076 \\
\hline Working $\mathrm{pH}$ range & $2.5-5$ & $6-10$ & $2.5-5$ & $6-10$ \\
\hline Response time (seconds) & 13 & 17 & 20 & 21 \\
\hline Lifetime (days) & 63 & 49 & 49 & 49 \\
\hline Accuracy $^{\mathrm{b}}(\mathrm{R} \%)$ & 100.95 & 99.60 & 98.48 & 101.27 \\
\hline Repeatability $^{\mathrm{c}}$ (RSD\%) & 1.53 & 0.31 & 0.98 & 0.44 \\
\hline $\begin{array}{l}\text { Intermediate precision }{ }^{\mathrm{d}} \text { (RSD } \\
\%)\end{array}$ & 1.90 & 0.86 & 1.05 & 1.19 \\
\hline
\end{tabular}

${ }^{\mathrm{a}}$.Lod $3.3 \mathrm{SD}$ of intercept/slope, and $\mathrm{LOQ}=10 * \mathrm{SD} /$ slope. ${ }^{\mathrm{b}}$ Average of three determinations. ${ }^{\mathrm{c}}$ Repeatability: the intraday precision $(n=3 \times 3)$ and average of three concentrations $\left(5 * 10^{-5}, 5 * 10^{-4}\right.$, and $\left.5 * 10^{-3} \mathrm{M}\right)$ were repeated three times within the day. ${ }^{\mathrm{d}}$ Intermediate precision: the interday precision $(n=3 \times 3)$ and average of three concentrations $\left(5 * 10^{-5}, 5 * 10^{-4}\right.$, and $\left.5 * 10^{-3} \mathrm{M}\right)$ were repeated three times on two consecutive days.

TABLE 2: Effect of the coating solution composition $\%(w / w)$ of DES- and MON-coated graphite sensors.

\begin{tabular}{|c|c|c|c|c|c|c|c|}
\hline & & Comp & & & & Slone & $\mathrm{ade}^{-1}$ \\
\hline & $\mathrm{PVC} \%$ & DBP\% & & & Linearity (MI) & Siope 1 ( & acte \\
\hline & 47.75 & 47.75 & & & $5 \times 10^{-4}-1 \times 10^{-2}$ & & \\
\hline & 45 & 45 & & & $5 \times 10^{-5}-1 \times 10^{-2}$ & & \\
\hline DFS RNK soncor & 60 & 30 & & & $5 \times 10^{-5}-1 \times 10^{-2}$ & & \\
\hline DES.RNK sensor & $30^{*}$ & 60 & & & $5 \times 10^{-5}-1 \times 10^{-2}$ & & \\
\hline & 40 & 40 & & & $5 \times 10^{-5}-1 \times 10^{-2}$ & & \\
\hline & 35 & 45 & & & $5 \times 10^{-5}-1 \times 10^{-2}$ & & \\
\hline & 47.5 & 47.5 & & & $5 \times 10^{-5}-1 \times 10^{-2}$ & & \\
\hline & 45 & 45 & & & $1 \times 10^{-5}-1 \times 10^{-2}$ & & \\
\hline & 60 & 30 & & & $1 \times 10^{-5}-1 \times 10^{-2}$ & & \\
\hline MON-Cd. sensor & $30^{*}$ & 60 & & & $1 \times 10^{-5}-1 \times 10^{-2}$ & & \\
\hline & 40 & 40 & & & $1 \times 10^{-5}-1 \times 10^{-2}$ & & \\
\hline & 35 & 45 & & & $1 \times 10^{-5}-1 \times 10^{-2}$ & & \\
\hline & 30 & 60 & 5 & 5 & $1 \times 10^{-4}-1 \times 10^{-2}$ & -23.12 & 24.23 \\
\hline & 40 & 40 & 10 & 10 & $5 \times 10^{-5}-1 \times 10^{-2}$ & -27.56 & 28.50 \\
\hline Combined sensor & $24^{*}$ & 48 & 10 & 10 & $1 \times 10^{-5}-1 \times 10^{-2}$ & -29.16 & 29.79 \\
\hline & 45 & 35 & 10 & 10 & $1 \times 10^{-5}-1 \times 10^{-2}$ & -28.76 & 29.42 \\
\hline
\end{tabular}

*Best percentages of the components which gave the best sensor's characteristics.

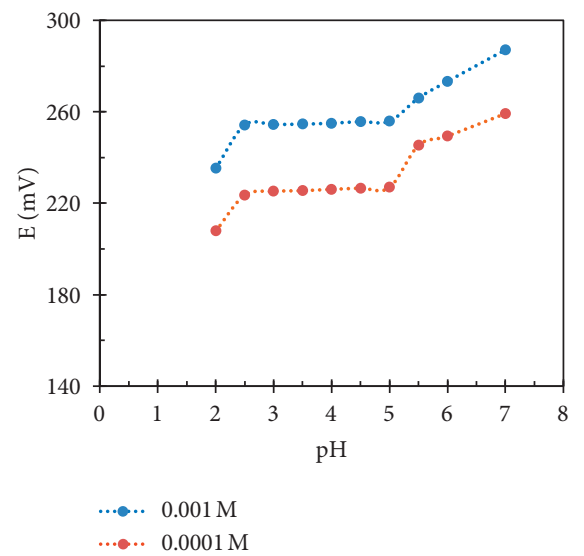

FIGURE 3: Effect of $\mathrm{pH}$ on potentiometric response for the DES.RNK sensor.
3.6. Method Validation. We have validated the proposed method, according to the ICH rules [36] as following.

3.6.1. Linearity. To estimate the linearity range of the constructed sensors, the potentials of a standard series consisting of 10 concentrations ranging between $1 \times 10^{-6} \mathrm{M}$ and $1 \times 10^{-1} \mathrm{M}$ were measured to get the regression equation for each sensor; the linearity range was found to be $5 \times 10^{-5}-1 \times 10^{-2} \mathrm{M}$ and $1 \times 10^{-5}-1 \times 10^{-2} \mathrm{M}$ for the DES sensor and MON sensor respectively, as shown in Figures 9 and 10 . When we used the combined sensor, we noticed a wider linearity range for both drugs $1 \times 10^{-5}-1 \times 10^{-2} \mathrm{M}$ and $5 \times 10^{-6}-1 \times 10^{-2} \mathrm{M}$ for DES and MON, respectively, that maybe due to the higher percentage of the electroactive material in membrane composition. 


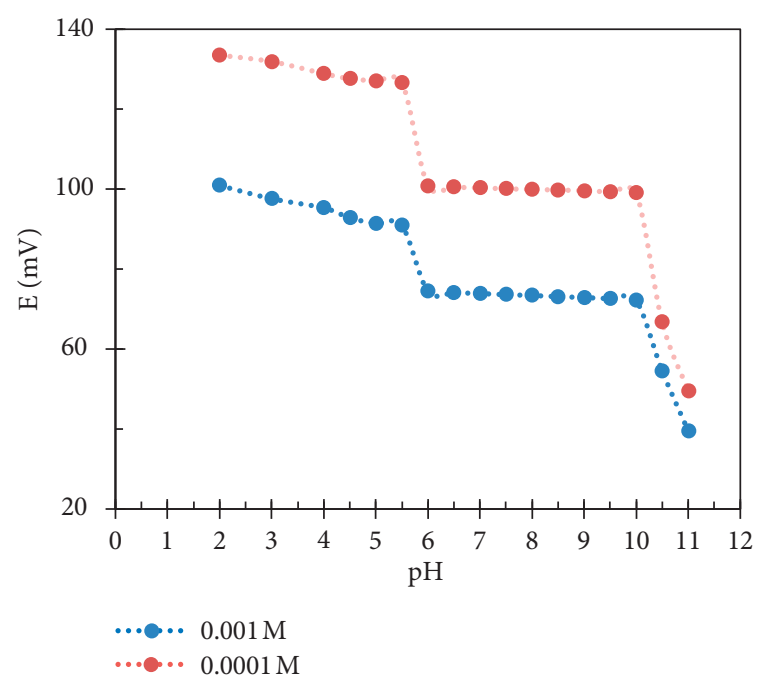

Figure 4: Effect of $\mathrm{pH}$ on potentiometric response for the MON.Cd. sensor.

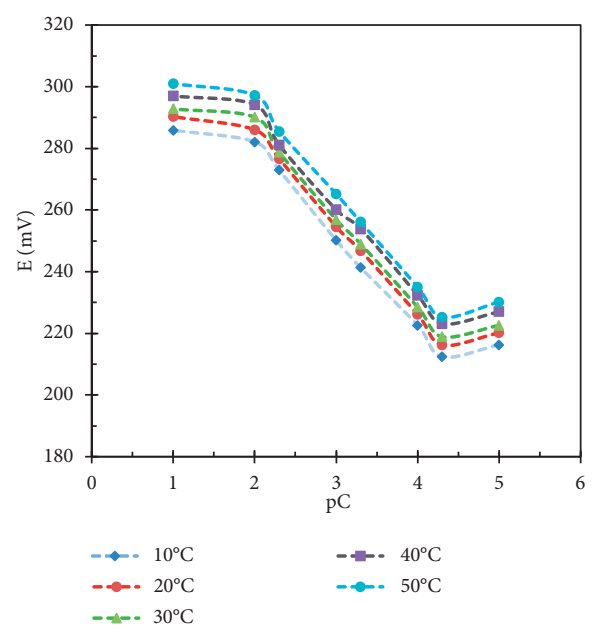

Figure 5: Effect of temperature on DES.RNK sensor response.

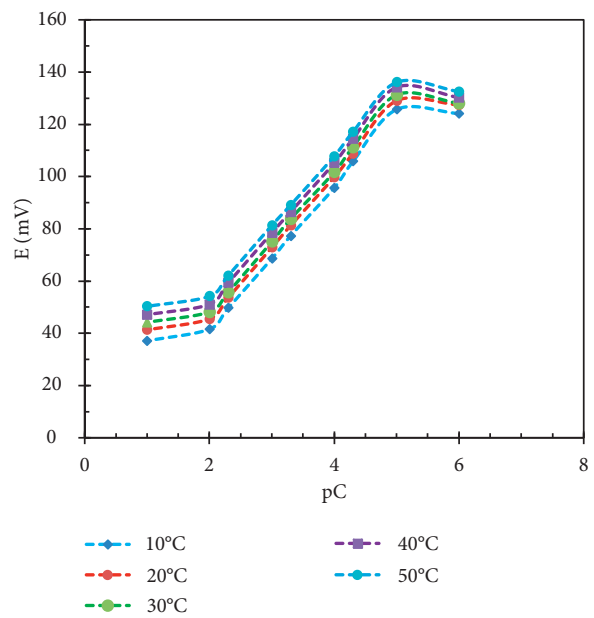

FIGURE 6: Effect of temperature on MON.Cd. sensor response.
TABLE 3: Selectivity coefficients of DES- and MON-coated graphite sensors.

\begin{tabular}{lcccc}
\hline \multirow{2}{*}{ Interfering } & DES.RNK & MON.Cd. & \multicolumn{2}{c}{ Combined sensor } \\
& sensor & sensor & DES & MON \\
& $\mathrm{K}_{\mathrm{DES}, \mathrm{B}}$ & $\mathrm{K}_{\mathrm{MON}, \mathrm{B}}$ & $\mathrm{K}_{\mathrm{DES}, \mathrm{B}}$ & $\mathrm{K}_{\mathrm{MON}, \mathrm{B}}$ \\
\hline $\mathrm{K}^{+}$ & $3.7 \times 10^{-3}$ & $1.6 \times 10^{-3}$ & $3.3 \times 10^{-3}$ & $1.4 \times 10^{-3}$ \\
$\mathrm{Na}^{+}$ & $4.4 \times 10^{-3}$ & $3.1 \times 10^{-3}$ & $4.1 \times 10^{-3}$ & $3.2 \times 10^{-3}$ \\
$\mathrm{NH}^{+}$ & $2.6 \times 10^{-3}$ & $7.8 \times 10^{-3}$ & $2.2 \times 10^{-3}$ & $7.5 \times 10^{-3}$ \\
$\mathrm{Ca}^{2+}$ & $1.6 \times 10^{-3}$ & $3.9 \times 10^{-3}$ & $1.3 \times 10^{-3}$ & $3.6 \times 10^{-3}$ \\
$\mathrm{Mg}^{2+}$ & $1.8 \times 10^{-3}$ & $8.1 \times 10^{-3}$ & $1.6 \times 10^{-3}$ & $8.3 \times 10^{-3}$ \\
$\mathrm{Mn}^{2+}$ & $2.1 \times 10^{-3}$ & $8.6 \times 10^{-3}$ & $2.2 \times 10^{-3}$ & $8.7 \times 10^{-3}$ \\
$\mathrm{Cu}^{2+}$ & $4.5 \times 10^{-3}$ & $7.4 \times 10^{-3}$ & $4.4 \times 10^{-3}$ & $7.6 \times 10^{-3}$ \\
$\mathrm{Fe}^{2+}$ & $5.1 \times 10^{-3}$ & $6.8 \times 10^{-3}$ & $5.3 \times 10^{-3}$ & $6.6 \times 10^{-3}$ \\
$\mathrm{Cd}^{2+}$ & $3.9 \times 10^{-3}$ & $6.5 \times 10^{-2}$ & $4.1 \times 10^{-3}$ & $6.2 \times 10^{-2}$ \\
$\mathrm{Avicel}^{2}$ & $1.9 \times 10^{-3}$ & $7.1 \times 10^{-3}$ & $1.5 \times 10^{-3}$ & $7.3 \times 10^{-3}$ \\
Mg stearate & $2.3 \times 10^{-3}$ & $4.5 \times 10^{-3}$ & $1.9 \times 10^{-3}$ & $4.3 \times 10^{-3}$ \\
Dextrose & $3.3 \times 10^{-3}$ & $6.2 \times 10^{-3}$ & $3.1 \times 10^{-3}$ & $5.8 \times 10^{-3}$ \\
Desloratadine & - & $1.1 \times 10^{-2}$ & - & $1.3 \times 10^{-2}$ \\
Montelukast & $1.5 \times 10^{-2}$ & - & $1.2 \times 10^{-2}$ & - \\
\hline
\end{tabular}

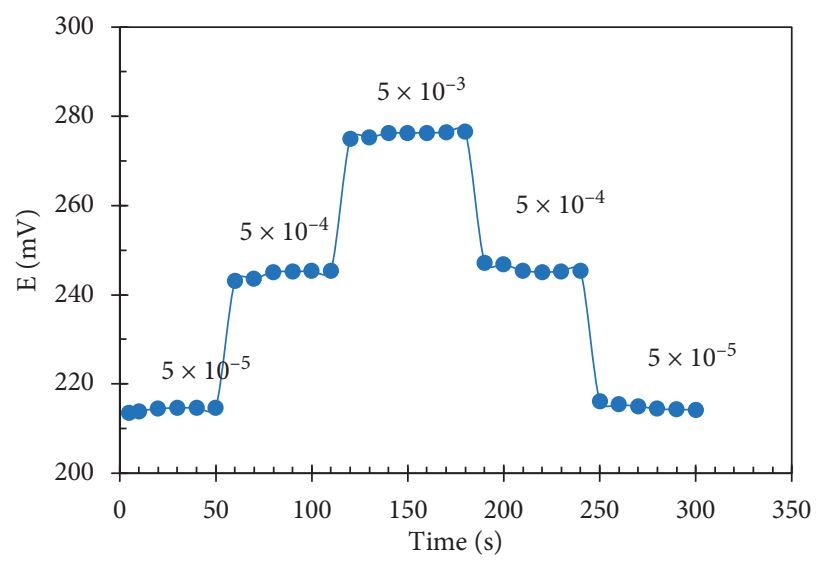

FIgURE 7: Dynamic response time and reversibility of DES.RNK electrode.

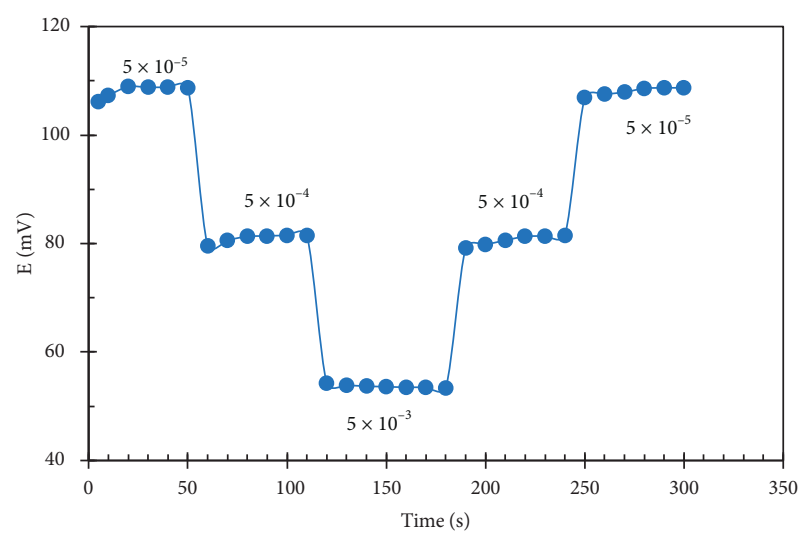

FIgure 8: Dynamic response time and reversibility of MON.Cd. electrode.

3.6.2. Accuracy and Precision. The method has been proven to be accurate by calculating the recovery values for 3 concentrations, which were chosen within the linearity 


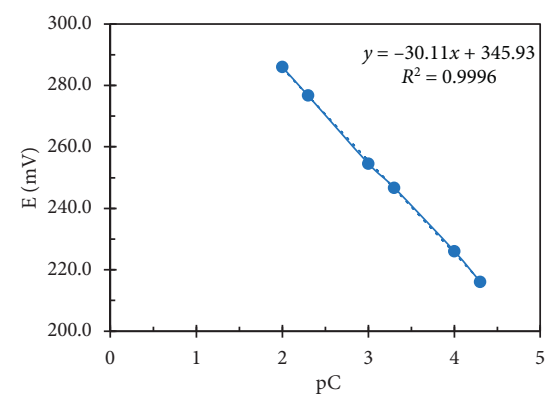

Figure 9: Potentiometric linearity profile of DES.RNK graphite sensor.

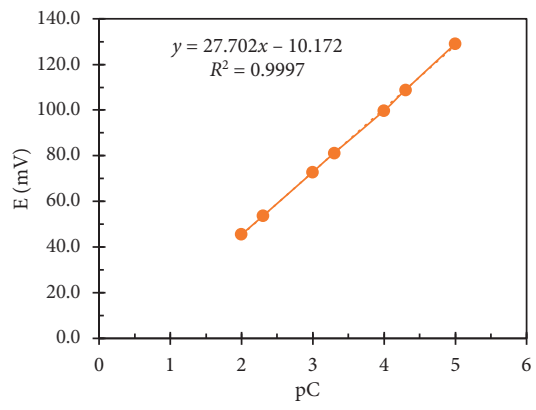

FIGURE 10: Potentiometric linearity profile of MON.Cd. graphite sensor.

TABLE 4: Determination of DES and MON in laboratory prepared mixtures by the proposed sensors.

\begin{tabular}{|c|c|c|c|c|c|}
\hline \multicolumn{2}{|c|}{ Ratio } & \multicolumn{4}{|c|}{ Recovery \% } \\
\hline \multirow{2}{*}{ DES } & \multirow{2}{*}{ MON } & \multirow{2}{*}{ DES.RNK sensor } & \multirow{2}{*}{ MON.Cd. sensor } & \multicolumn{2}{|c|}{ Combined sensor } \\
\hline & & & & DES & MON \\
\hline 1 & 1 & 100.65 & 100.98 & 100.54 & 100.92 \\
\hline 1 & 2 & 99.93 & 100.56 & 99.89 & 99.75 \\
\hline 1 & 5 & 98.23 & 100.23 & 98.11 & 100.05 \\
\hline 2 & 1 & 100.18 & 99.34 & 100.08 & 99.22 \\
\hline 5 & 1 & 100.87 & 98.92 & 100.76 & 100.79 \\
\hline \multicolumn{2}{|c|}{ Mean \pm SD } & $99.97 \pm 1.04$ & $100.01 \pm 0.86$ & $99.71 \pm 1.30$ & $100.15 \pm 0.71$ \\
\hline
\end{tabular}

TABLE 5: Determination of DES and MON in pharmaceutical preparations by the constructed sensors and reported methods.

\begin{tabular}{|c|c|c|c|c|c|}
\hline Commercial name & Composition & Amount found, $\mathrm{mg}^{\mathrm{a}}$ & $\mathrm{R} \% \pm \mathrm{SD}$ & $t$ value $^{\mathrm{b}}$ & $F$ value $^{\mathrm{c}}$ \\
\hline \multicolumn{6}{|l|}{ Sensor 1 DES.RNK } \\
\hline Aerius & Desloratadine, $5 \mathrm{mg}$ & 4.980 & $99.60 \pm 1.80$ & 1.77 & 1.41 \\
\hline Azmalir & Montelukast sodium, $10 \mathrm{mg}$ & - & - & - & - \\
\hline \multirow{2}{*}{ Desolate-M } & Desloratadine, $5 \mathrm{mg}$ & 4.993 & $99.87 \pm 1.22$ & 0.89 & 1.55 \\
\hline & Montelukast sodium, $10 \mathrm{mg}$ & - & - & - & - \\
\hline \multicolumn{6}{|l|}{ Sensor 2 MON.Cd. } \\
\hline Aerius & Desloratadine, $5 \mathrm{mg}$ & - & - & - & - \\
\hline Azmalir & Montelukast sodium, $10 \mathrm{mg}$ & 9.990 & $99.90 \pm 0.85$ & 0.30 & 2.34 \\
\hline \multirow{2}{*}{ Desolate-M } & Desloratadine, $5 \mathrm{mg}$ & - & - & - & - \\
\hline & Montelukast sodium, $10 \mathrm{mg}$ & 9.916 & $99.16 \pm 0.61$ & .0 .92 & 1.00 \\
\hline \multicolumn{6}{|c|}{ Sensor 3 combined sensor } \\
\hline Aerius & Desloratadine, $5 \mathrm{mg}$ & 4.990 & $99.80 \pm 1.60$ & 1.04 & 1.11 \\
\hline Azmalir & Montelukast sodium, $10 \mathrm{mg}$ & 9.960 & $99.60 \pm 1.15$ & 0.60 & 1.27 \\
\hline \multirow{2}{*}{ Desolate-M } & Desloratadine, $5 \mathrm{mg}$ & 5.020 & $100.40 \pm 1.05$ & 0.76 & 0.52 \\
\hline & Montelukast sodium, $10 \mathrm{mg}$ & 9.920 & $99.20 \pm 0.92$ & 0.83 & 2.27 \\
\hline
\end{tabular}

${ }^{a}$ Average of 3 replicates; ${ }^{\mathrm{b}} t$ critical $4.302(0.05) ;{ }^{\mathrm{c}} f$ critical $19(0.05) ; n=3$. 
range by direct potentiometric measurements. Each concentration was measured in triplicate, and the average recovery values were $100.95 \%$ and $99.60 \%$ for sensor 1 and sensor 2, respectively, that approved the accuracy of the proposed method.

The precision of the method was verified at two levels (repeatability and intermediate precision) and was performed by triplicate determination for 3 concentrations which were measured on the same day for the interday level and on 3 different days for the intraday level. The values of $\mathrm{RSD}$, which were $<2 \%$, indicate the precision of the method, as given in Table 1.

3.6.3. Specificity. The specificity of the proposed method was confirmed by preparing laboratory mixtures of desloratadine and montelukast sodium at different ratios. The recovery for each drug was determined by its proposed sensor in presence of the other drugs. The recovery values which are given in Table 4 indicate the specificity of the method.

3.6.4. Pharmaceutical Applications. The proposed method was successfully applied to determine desloratadine and montelukast sodium in their pharmaceutical preparations as single dosage and as a combined dosage form (desolate- $\mathrm{M}$ ), without any separation depending on the effective $\mathrm{pH}$ range for each electrode. The recovery outcomes which are given in Table 5 reveal the ability of the proposed sensors to be used for direct potentiometric determination of drugs in its pharmaceutical forms without any interference of the excipients. The statistical tests ( $t$ test and $F$ test) were applied to confirm that the results which we got do not differ from the results which were reported in a reference HPLC method [19] that denote the accuracy and precision of the proposed method.

\section{Conclusions}

Summing up the results, it can be concluded that the proposed pencil graphite sensors can be used as a selective, sensitive, and validated analytical technique for the potentiometric determination of desloratadine and montelukast in their combined dosage form without any separation step. This research was the first trial for electrochemical determination of this combination. The proposed method could compete with the complicated methods which were reported to determine this combination, being simpler, faster, ecofriendly, moreover the wide linear range and long lifetime of the electrodes that save time and the efforts of the analysis process.
Abbreviations
DES: Desloratadine
MON: Montelukast sodium
RNK: Ammonium reineckate
Cd: Cadmium chloride
PVC: Polyvinyl chloride
DBP: Dibutyl phthalate

THF: Tetrahydrofuran

ICH: The International Council for Harmonization of Technical Requirements for Pharmaceuticals for Human Use.

\section{Data Availability}

The datasets used and analysed during the current study are available from the corresponding author upon request.

\section{Conflicts of Interest}

The authors declare that they have no conflicts of interest.

\section{Authors' Contributions}

A.A. Sakur conceived and designed the experiments. D. Nashed performed the experiments and wrote the article. I. Noureldin analysed and interpreted the data. All authors read and approved the final draft of the article.

\section{References}

[1] J. Foucquier and M. Guedj, "Analysis of drug combinations: current methodological landscape," Pharmacology Research \& Perspectives, vol. 3, no. 3, Article ID e00149, 2015.

[2] G. G. Mohamed, F. M. A. Attia, and N. S. Ibrahim, "Development and validation of spectrophotometric and HPLC methods for the determination of desloratadine in tablets and syrup," Journal of Pharmacy Research, vol. 5, pp. 2799-2805, 2012.

[3] G. G. Mohamed, F. M. Abou Attia, N. S. Ismail, and N. S. Ibrahim, "Analytical uses of charge-transfer complexes: determination of dosage forms of desloratadine," Acta Pharmaceutica Scienica, vol. 53, pp. 89-98, 2011.

[4] The United States Pharmacopiea, The United States Pharmacopiea, Sep, United States Pharmacopeial Convention, Rockvile, ML, USA, 2016.

[5] F. Horak, U. P. Stübner, R. Zieglmayer, A. G. Harris, and A. G. Harris, "Effect of desloratadine versus placebo on nasal airflow and subjective measures of nasal obstruction in subjects with grass pollen-induced allergic rhinitis in an allergen-exposure unit," Journal of Allergy and Clinical Immunology, vol. 109, no. 6, pp. 956-961, 2002.

[6] L. Liu, M. Qi, P. Wang, and H. Li, "High-performance liquid chromatographic method for the bioequivalence evaluation of desloratadine fumarate tablets in dogs," Journal of Pharmaceutical and Biomedical Analysis, vol. 34, no. 5, pp. 1013-1019, 2004.

[7] C. Chandra Sekhar, U. Viplava Prasad, and K. Naresh Kumar, "New spectrophotometric method for the determination of desloratadine in pharmaceutical formulations," International Journal of Chemical Science, vol. 10, pp. 2067-2072, 2012.

[8] D. Sri Vidya, M. Siva Prasad, M. Vishnu Priya, K. Roja, and N. Y. Sreedhar, "Voltammetric determination of desloratadine in pharmaceutical and human urine samples using glassy carbon electrode," International Journal of Pharmacy and Pharmaceutial Science, vol. 6, no. 10, pp. 119-122, 2014.

[9] S. C. Sweetman, Ed., Journal of Medical Library Associationp. 1240, 37th edition, Pharmaceutical Press, London, UK, 2011.

[10] A. K. Shakya, T. A. Arafat, N. M. Hakooz, A. N. Abuawwad, H. Al-Hroub, and M. Melhim, "High-performance liquid 
chromatographic determination of montelukast sodium in human plasma: application to bioequivalence study," Acta Chromatographica, vol. 26, no. 3, pp. 457-472, 2014.

[11] B. Chauhan, S. Rani, M. Nivsarkar, and H. Padh, "A new liquid-liquid extraction method for determination of montelukast in small volume human plasma samples using HPLC with fluorescence detector," Indian Journal of Pharmaceutical Sciences, vol. 68, no. 4, p. 517, 2006.

[12] S. Muralidharan, L. Jia Qi, L. Ting Yi et al., "Newly developed and validated method of montelukast sodium estimation in tablet dosage form by ultraviolet spectroscopy and reverse phase-high performance liquid chromatography," Pharmacology, Toxicology and Biomedical Reports, vol. 2, no. 2, pp. 27-30, 2016.

[13] P. Kurra and P. S. Babu, "Validated UV spectroscopic method for estimation of montelukast," International Journal of Pharmacy and Medical Science, vol. 1, pp. 104-111, 2012.

[14] A. A. Sakur, D. Nashed, and I. Noureldin, "Novel pencil graphite electrode for the determination of montelukast sodium in its pure and pharmaceutical dosage forms key words," Research Journal of Pharmacy and Technology, vol. 14. , 2021 In press.

[15] A. T. Soudi, O. G. Hussein, E. S. Elzanfaly, H. E. Zaazaa, and M. Abdelkawy, "Potentiometric method to determine montelukast sodium in its tablets with in-line monitoring of its dissolution behaviour," Analytical and Bioanalytical Electrochemistry, vol. 12, pp. 502-516, 2020.

[16] D. Nashed, I. Noureldin, and A. A. Sakur, "New pencil graphite electrodes for potentiometric determination of fexofenadine hydrochloride and montelukast sodium in their pure, synthetic mixtures, and combined dosage form," $B M C$ Chemistry, vol. 14, no. 1, 2020.

[17] B. E. Davis, D. C. Todd, D. W. Cockcroft, D. W. Cockcroft, and C. Frcp, "Effect of combined montelukast and desloratadine on the early asthmatic response to inhaled allergen," Journal of Allergy and Clinical Immunology, vol. 116, no. 4, pp. 768-772, 2005.

[18] R. Challa and N. V. S. Naidu, "Development and validation of stability indicating RP-HPLC method for simultaneous determination of desloratadine and montelukast sodium in pharmaceutical dosage form," Journal of Pharmscy Analitical Research, vol. 5, pp. 294-309, 2016.

[19] V. R. Chhatrala and J. Patel, "Simultaneous estimation of montelukast sodium and desloratadine by RP-HPLC in their marketed formulation," International Journal of Chemistry andTechnology, vol. 4, pp. 1402-1407, 2012.

[20] E. N. Kumar, D. Sireesha, V. Bakshi, M. A. Haque, and S. Harshini, "Simultaneous estimation of desloratadine and montelukast in combined pharmaceutical dosage form by UV spectroscopy," International Journal of Innovative Pharmceutical Science and Research, vol. 2, pp. 2765-2772, 2014.

[21] F. A. Ibrahim, N. El-enany, R. N. El-shaheny, and I. E. Mikhail, "Simultaneous determination of desloratadine and montelukast sodium using second-derivative synchronous fluorescence spectrometry enhanced by an organized medium with applications to tablets and human plasma," Luminescence, vol. 30, no. 4, pp. 485-494, 2015.

[22] A. Bratovcic, A. Odobasic, and S. Catic, "The advantages of the use of ion- selective potentiometry in relation to UV/VIS spectroscopy the advantages of the use of Ion- selective potentiometry in relation to UV/VIS spectroscopy," Agriculturae Conspectus Science, vol. 74, pp. 139-142, 2009.

[23] O. Mansour, D. Nashed, and A. A. Sakur, "Determination of clopidogrel bisulphate using drug selective membranes,"
Research Journal of Pharmacy and Technology, vol. 11, no. 5, pp. 2017-2021, 2018.

[24] H. Dabbeet, A. A. Sakur, and I. Noureldin, "Novel drug selective sensors for simultaneous potentiometric determination of both ciprofloxacin and metronidazole in pure form and pharmaceutical formulations," Research Journal of Pharmacy and Technology, vol. 12, pp. 3377-3384, 2019.

[25] A. A. Sakur, H. Dabbeet, and I. Noureldin, "Simultaneous potentiometric determination of ciprofloxacin- meteronidazole-minocycline in triad combination using novel drug selective sensor," Journal of Global Trends Pharmaceutical and Science, vol. 11, pp. 8255-8271, 2020.

[26] A. A. Sakur, H. A. Dabbeet, and I. Noureldin, "Novel drug selective polystyrene membrane for simultaneous potentiometric determination of ciprofloxacin and tinidazole in pure form and pharmaceutical formulations," Research Journal of Pharmacy and Technology, vol. 13, no. 12, pp. 5963-5971, 2020.

[27] R. W. Cattarall and F. Henry, "Coated wire ion- selective electrode," Analytical Chemistry, vol. 43, pp. 1905-1906, 1971.

[28] A. A. Sakur, S. Bassmajei, and H. A. Dabbeet, "Novel moxifloxacin ion selective electrodes for potentiometric determination of moxifloxacin in pure form and pharmaceutical formulations," International Journal of Acadamic Scientific Research, vol. 3, pp. 66-75, 2015.

[29] M. Haroun, D. Nashed, and A. A. Sakur, "New electrochemical methods for the determination of Prasugrel using drug selective membranes," International Journal Acadamic Scientific Research, vol. 5, pp. 30-36, 2017.

[30] A. A. Sakur, D. Nashed, M. Haroun, and I. Noureldin, "Determination of prasugrel hydrochloride in bulk and pharmaceutical formulation using new ion selective electrodes," Research Journal of Pharmacy and Technology, vol. 11, no. 2, pp. 631-636, 2018.

[31] H. Mandil, A. A. Sakur, and B. Nasser, "New ion selective electrode for potentiometric determination of gatifloxacin in pure form and pharmaceutical formulations," International Journal of Pharmacy and Pharmaceutical Science, vol. 5, 2013.

[32] F. I. Khattab, N. K. Ramadan, M. A. Hegazy, and N. S. Ghoniem, "Microsized graphite sensors for potentiometric determination of metronidazole and spiramycin," Portugaliae Electrochimica Acta, vol. 29, no. 2, pp. 79-90, 2011.

[33] M. M. Khalil, Y. M. Issa, and A. G. Mohamed, "Construction and performance characterization of ion-selective electrodes for potentiometric determination of paroxetine hydrochloride in pharmaceutical preparations and biological fluids," Electroanalysis, vol. 26, no. 12, pp. 2789-2800, 2014.

[34] Y. Umezawa, K. Umezawa, and H. Sato, "Selectivity coefficients for ion-selective electrodes: recommended methods for reporting KA, bpot values (technical report)," Pure and Applied Chemistry, vol. 67, no. 3, pp. 507-518, 1995.

[35] M. Giahi, H. Aghaie, and K. Mehdizadeh, "Design and manufacture of silver-selective electrode based on singlewalled carbon nanotubes design and manufacture of silverselective electrode based on single-walled carbon nanotubes, orient," Journal of Chemistry, vol. 31, pp. 703-708, 2015.

[36] International Conference on Harmonization (ICH), Validation of Analytical Procedures: Text and Methodology, Q2 (R1) IFPMA, Geneva, Switzerland, 2005. 